\title{
Surgical Resection of Giant Pheochromocytomas Arising Behind the Retrohepatic Inferior Vena Cava
}

\author{
YUJI SOEJIMA, TOMOHARU YOSHIZUMI, TORU IKEGAMI, \\ NORIFUMI HARIMOTO, SHINJI ITO, NOBORU HARADA, TAKASHI MOTOMURA, \\ AKIHISA NAGATSU, TETSUO IKEDA and YOSHIHIKO MAEHARA
}

Departments of Surgery and Science, Graduate School of Medical Sciences, Kyushu University, Fukuoka, Japan

\begin{abstract}
Background: Surgical resection of retrocaval giant pheochromocytomas (PCs) and of extra-adrenal paragangliomas (EAPs) is a technically challenging procedure but data on these procedures are scarce. The purpose of this study was to present and evaluate our surgical results for such tumors. Patients and Methods: We retrospectively analyzed four consecutive patients who had surgery for giant retro-caval PCs and EAPs in terms of surgical technique, resectability of the tumor, requirement for bypass, and postoperative complications. A laparoscopic approach was not feasible in all cases because of the undesirable location of the tumors. Results: In all cases, the liver was completely mobilized from the right side to expose and control the supra-and infra-hepatic inferior vena cava. Resection of the tumors was feasible for all patients with a minimum titration of blood pressure during surgery. None of the cases required venous bypass. In the patient who had the largest tumor, the infra-hepatic inferior vena cava was transected temporally to obtain direct and maximum exposure of the tumor. None of the patients have had any postoperative complications and all are currently alive without recurrence and use of antihypertensive drugs. Conclusion: Resection of retrocaval giant PCs and EAPs is a safe procedure. Temporal transection of the infra-hepatic inferior vena cava can offer excellent exposure, especially for an extremely large tumor, without compromising hepatic and systemic hemodynamics.
\end{abstract}

Pheochromocytomas (PCs) are defined as tumors arising from chromaffin cells of the adrenal medulla (1). Closely

Correspondence to: Yoshihiko Maehara, MD, Ph.D, FACS and Yuji Soejima, MD, Ph.D., FACS, Departments of Surgery and Science, Graduate School of Medical Sciences, Kyushu University, Fukuoka 812-8582, Japan. Tel: +81 926425466, Fax: +81 926425482, e-mail: ysoejima@surg2.med.kyushu-u.ac.jp

Key Words: Pheochromocytoma, paraganglioma, temporal transection, inferior vena cava, liver transplantation. related tumors arising from extra-adrenal sympathetic and parasympathetic paraganglia are classified as extra-adrenal paragangliomas (EAPs) (1). EAPs can be found from the base of the skull to the pelvis and are classified into four basic groups according to their anatomical site: branchiomeric, intravagal, aorticosympathetic and visceral autonomic (2). The retroperitoneum is the most common site of occurrence. Fifteen percent of PCs and $57.1 \%$ of EAPs are functional tumors that produce various types of catecholamines $(3,4)$. They are associated with serious mortality and morbidity because of the potential effect of catecholamines on different organs, especially the cardiovascular system. Moreover, malignant tumors comprise $10 \%$ of these tumor types (4). PCs and EAPs often involve or invade the inferior vena cava (IVC) $(5,6)$. Slycke et al. retrospectively reviewed 51 patients with EAPs and described the incidence, characteristics and outcome and found that EAPs recur at the rate of $15 \%$ at 5 years and $23 \%$ at 10 years (7); therefore, long-term follow-up using imaging studies is imperative. Complete surgical resection of the tumor is the only option for cure of these tumors, but is associated with potential risks for hypertension crises and serious arrhythmia because of the massive release of catecholamines. Currently, laparoscopic surgery is the most preferred treatment option (8). However, these tumors sometimes become gigantic and arise from very difficult areas, such as the retroperitoneum behind the retrohepatic IVC (also referred to as the retrocaval region), which makes surgical treatment including the laparoscopic approach extremely difficult. To our knowledge, there have been no reports on surgical management for such types of tumors.

The reasons why surgery around this region is very difficult include the following. Firstly, the right lobe of the liver and the retrohepatic IVC lie on the tumor (in a 'piggyback' fashion) and complete exposure of the tumor is very difficult. Secondly, surgical manipulation of the tumors should be minimal in order to avoid hypertensive crises during surgery. Therefore, meticulous dissection without mobilization and complete devascularization of the tumor are 
imperative in order to achieve this goal. Techniques for liver surgery, especially for liver transplantation, are essential to overcome these problems (9).

In the present study, we retrospectively reviewed four consecutive cases of retrocaval giant PGs and ESPs that were successfully treated with standardized management and surgical procedures. Technical details of the procedures are discussed. Furthermore, a new technique that might facilitate maximum exposure of the tumor is presented.

\section{Patients and Methods}

Between December 2006 and March 2010, four patients with giant retrocaval tumors presented with large abdominal tumors accompanied by acute and chronic symptoms including hypertension $(n=4)$, headache $(n=3)$, chest pain $(n=2)$, back pain $(n=1)$ and abdominal pain $(n=1)$, palpitation $(n=2)$ and sweating $(n=2)$. All patients had been treated with antihypertensive drugs including alpha-blockers. There were two male and two female patients. The mean age of the patients was 43.5 years (range $=20-66$ years). The mean preoperative tumor size was $8.8 \mathrm{~cm}$ (range $=5.5$ $16.7 \mathrm{~cm}$ ). The mean preoperative serum and urine catecholamine levels were elevated as follows: urine fractionated epinephrine $=316.8 \mu \mathrm{g} / \mathrm{ml} \quad$ (normal range $=3.4-26.9 \mu \mathrm{g} / \mathrm{ml}$ ); norepinephrine $=992.9 \mu \mathrm{g} / \mathrm{ml}$ (normal range $=48.6-168.4 \mu \mathrm{g} / \mathrm{ml}$ ); dopamine $=759.7 \mu \mathrm{g} / \mathrm{ml}$ (normal range $=365.0-961.5 \mu \mathrm{g} / \mathrm{ml}$ ); and vanillylmandelic acid $=27.3 \mu \mathrm{g} / \mathrm{mg}$ creatinine (normal range $=1.2-4.9$ $\mu \mathrm{g} / \mathrm{mg}$ creatinine). Plasma levels of epinephrine, norepinephrine, and dopamine were $485.5 \mathrm{pg} / \mathrm{ml}$ (normal range, $<100 \mathrm{pg} / \mathrm{ml}$ ), $1,832.5 \mu \mathrm{g} / \mathrm{ml}$ (normal range, $100-450 \mu \mathrm{g} / \mathrm{ml}$ ), and $19.8 \mathrm{pg} / \mathrm{ml}$ (normal range, $<20 \mathrm{pg} / \mathrm{ml}$ ), respectively. For all patients, diagnosis of PC or EAP was given on referral. All of the patients underwent elective surgical resection of the tumors after obtaining informed consent. Preoperative blood pressure was controlled within the normal range with antihypertensive drugs.

Surgical procedures and anesthesiological management of cases 1,2 , and 4 were basically similar. Briefly, the abdomen was entered by a bilateral subcostal incision with a midline extension. The right lobe of the liver, the duodenum and the right colon were mobilized until the retroperitoneal tumors and the IVC including the right renal vein were directly exposed. All tumors were found to be welldemarked and encapsulated. Firstly, the hepatoduodenal ligament was controlled with tape for Pringle's maneuver. The infrahepatic IVC was controlled with tape just proximal to the right renal vein conduit. The suprahepatic IVC was also controlled with tape. Multiple veins that drained from the tumor into the dorsal wall of the IVC were meticulously dissected and divided between ligatures and clips while the IVC was retracted ventrally. During the procedures, careful attention was paid not to touch or mobilize the tumor. However, several episodes of hypertensive surge were encountered during the dissection, for which antihypertensive drugs, including nicardipine, were effective. After completing devascularization, the tumors were freely mobilized and dissected using an electrocautery or vessel-sealing instrument (LigaSure Atlas $^{\mathrm{TM}}$; Valley Lab, Boulder, CO, USA). No drains were placed for these three cases.

In case 3, the tumor appeared too large to be exposed (Figure 1A and B). Therefore, the infrahepatic IVC was temporally transected after completing dissection between the tumor and the IVC (Figure
1C). Thereafter, the right lobe of the liver was mobilized to the left lobe with the transected IVC. The procedures provided excellent exposure of the tumor, which facilitated the dissection and free mobilization of the tumor, especially for the left side of the tumor. The total clamp time of the IVC was $63 \mathrm{~min}$. During the procedure, the hemodynamic parameters were consistently stable; therefore, a venovenous bypass was not necessary. After tumor excision and hemostasis of the dissected retroperitoneum, the transected IVC was reconstructed end-to-end with continuous 5-0 Prolene sutures and the IVC was reperfused (Figure 1D). The patient had no complications postoperatively and was discharged from the hospital at postoperative day 11 with normal blood pressure without drugs.

\section{Results}

A summary of the characteristics of the patients and the operative outcomes are shown in Table I. The mean operative time was $294 \mathrm{~min}$ and the mean blood loss was $552 \mathrm{ml}$. No patients required blood transfusion. The mean number of temporal interruptions of surgery due to hypertensive surge was three, for which antihypertensive drugs, including nicardipine, were given as needed. No patient required antihypertensive drugs immediately after surgery. There were no surgery-related complications. The mean hospital stay was 15.3 days. For all patients, the level of catecholamines returned to normal within 1-2 weeks.

Pathological examination of the four excised tumors revealed that all of the tumors were membrane-covered and appeared circumscribed. Local invasion was not evident. In cases 1-3, intact right adrenal glands abutted the tumor. However, in case 4 , the tumor appeared to derive from the adrenal gland. Microscopically, tumor cells consisted of cuboidal or polygonal basophilic cells, proliferating in a trabecular and solid fashion, and they stained positively for chromogranin A, which was compatible with the diagnosis of PCs or EAPs. Surgical margins were negative in all cases.

Currently, all patients are alive without recurrence and they are not on antihypertensive drugs, with a mean followup of 965 days.

\section{Discussion}

Standardized surgical management of giant PCs and EAPs has not been established because these are rare tumors. While $10 \%$ of EAPs arise from the retroperitoneum (4), they rarely arise from the retrocaval retroperitoneum, which makes surgical excision more challenging than usual retroperitoneal EAPs.

Prevention of massive catecholamine release from EAPs which lead to uncontrollable hypertensive crisis during surgery is the primary concern for surgeons as well as anesthesiologists. Therefore, the key to successful excision of functional EAPs is minimal dissection and mobilization of the tumor. Meticulous dissection and ligation of all of the blood vessels between the tumor and the IVC (feeding arteries and 

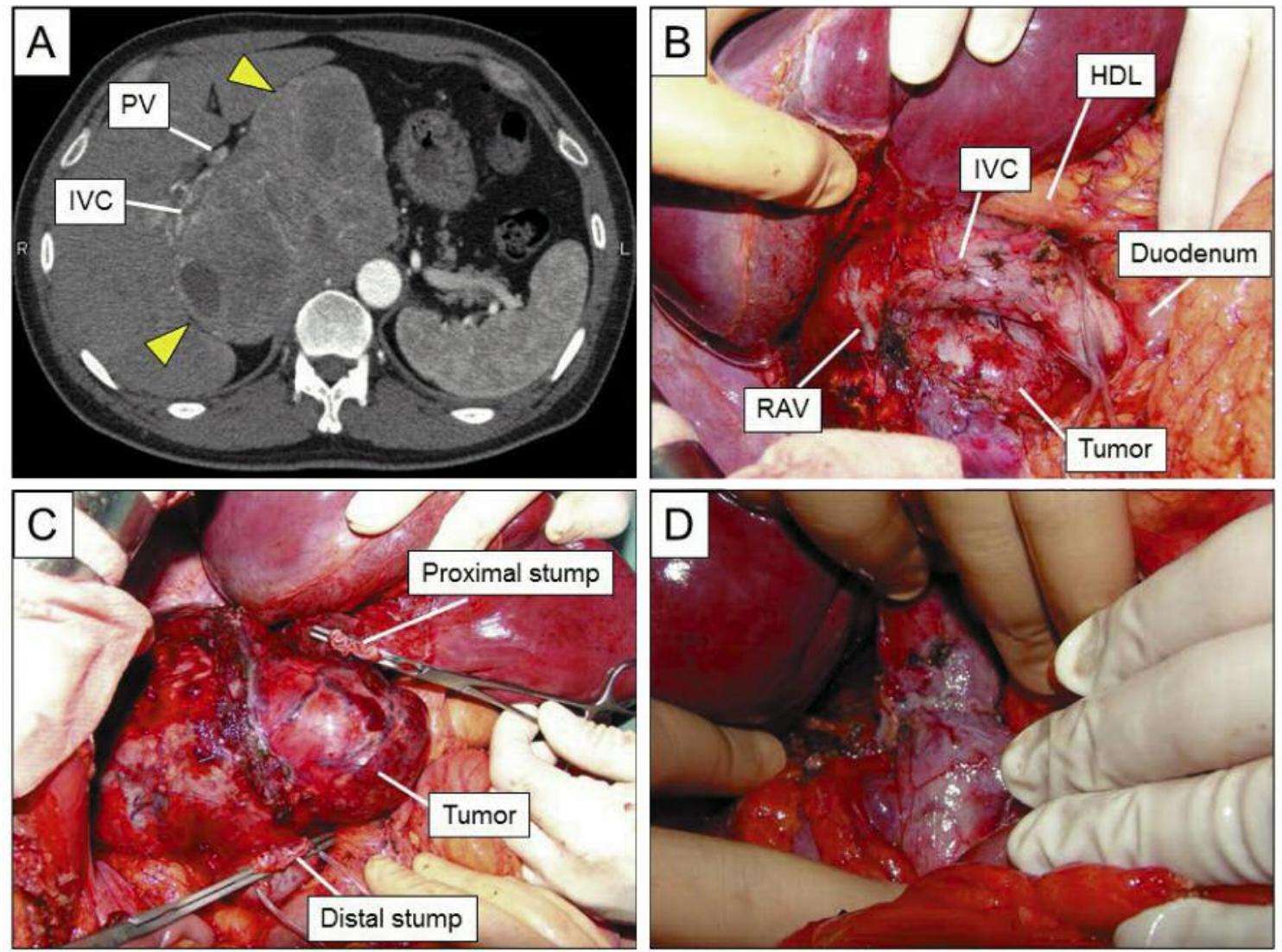

Figure 1. A: Preoperative computed tomographic scan of case 3. Note that the giant tumor (arrowheads) is located dorsal to the inferior vena cava (IVC). B: Intraoperative findings of the tumor. Note that the retrohepatic IVC 'piggy-backs' the tumor. After mobilizing the right side of the liver, the supra- and infrahepatic IVC were controlled with tape. Blood vessels between the IVC and the tumor were meticulously divided without mobilizing the tumor. C: An operative view after temporal transection of the supra-renal IVC and mobilization of the liver. Note the excellent exposure of the entire aspect of the tumor. The proximal and distal stumps of the transected IVC are also evident. D: The infrahepatic IVC after reconstruction. PV: Portal vein; HDL: hepato-duodenal ligament; RAV: right adrenal vein.

Table I. Characteristics of patients, tumors and operation. There were no complications or tumor recurrence in any of the patients.

\begin{tabular}{|c|c|c|c|c|c|c|c|c|c|c|}
\hline Case no. & $\begin{array}{l}\text { Age (years)/ } \\
\text { gender }\end{array}$ & Diagnosis & $\begin{array}{l}\text { Size } \\
(\mathrm{cm})\end{array}$ & $\begin{array}{l}\text { Operative } \\
\text { time (min) }\end{array}$ & $\begin{array}{l}\text { Blood loss } \\
\quad(\mathrm{ml})\end{array}$ & $\begin{array}{c}\text { Intraoperative HT } \\
\text { episodes, } \mathrm{n}^{*}\end{array}$ & TTIVC & $\begin{array}{l}\text { Hospital stay } \\
\quad \text { (days) }\end{array}$ & $\begin{array}{l}\text { Follow-up } \\
\text { (days) }\end{array}$ & Status \\
\hline 1 & $43 / \mathrm{F}$ & EAP & 6.0 & 210 & 70 & 2 & No & 11 & 1370 & Alive \\
\hline 2 & $20 / \mathrm{M}$ & EAP & 5.5 & 371 & 600 & 2 & No & 12 & 1308 & Alive \\
\hline 3 & $45 / \mathrm{M}$ & EAP & 16.7 & 372 & 1386 & 3 & Yes & 11 & 867 & Alive \\
\hline 4 & $66 / F$ & $\mathrm{PC}$ & 7.0 & 221 & 150 & 8 & No & 27 & 314 & Alive \\
\hline
\end{tabular}

F: Female; M: male; TTIVC, transient transection of the infra-hepatic IVC; EAP, extra-adrenal pheochromocytoma; PC, pheochromocytoma; HT, hypertension. *Defined as blood pressure more than $200 \mathrm{mmHg}$.

drainage veins of the tumor) is, therefore, an integral part of the procedure. Once devascularization is completed, the tumor should be able to be mobilized freely because catecholamine release from the tumor is blocked. For this reason, good exposure is a prerequisite to successful excision.
Temporal transection of the infra-hepatic IVC (TTIVC) is a new, simple and innovative technique that can offer maximum exposure of the tumor. It is expected to be very effective, especially for extremely large tumors such as that found in case 3 . However, in cases where the tumors are 
considered to be unresectable in situ, such as when tumor invasion to the IVC or the liver is evident, or the tumor is located between the IVC and the portal triad, ex vivo resection of the tumor and autotransplantation of the liver is an option (10). Increased surgical experience with liver transplantation has enabled surgeons to perform such a complex procedure $(11,12)$.

Using the TTIVC technique, we believe that venous bypass is not necessary because venous flow of the liver into the IVC is completely preserved during TTIVC. Deep venous thrombosis in the lower extremities may develop when the transection time is prolonged. We did not use systemic heparin during nor after TTIVC; however, no thrombotic complication developed. Based on our experience of liver transplantation, a 1-hour clamp of the IVC should not produce any problems associated with thrombosis.

Tominaga et al. reported a technique using IVC isolation and extracorporeal charcoal hemoperfusion (CHP) to avoid hypertensive crises due to massive catecholamine release (13). They found that IVC isolation with CHP removed $90 \%$ of released catecholamines during surgery, suggesting the usefulness of IVC isolation with CHP. This modality might be a promising tool to control hypertension but we currently believe that this procedure is only useful when the tumor is located caudal to the hepatic vein conduit. Therefore, this procedure was not used for the cases presented here.

In conclusion, resection of retrocaval giant PCs or EAPs is a safe and feasible procedure. TTIVC can offer excellent exposure without compromising hepatic and systemic hemodynamics and should be considered a standard procedure.

\section{Conflicts of Interest}

All Authors have no conflict of interest in regard to this study.

\section{References}

1 Tischler AS: Pheochromocytoma and extra-adrenal paraganglioma: update. Arch Pathol Lab Med 132: 1272-1284, 2008.

2 Disick GI and Palese MA: Extra-adrenal pheochromocytoma: diagnosis and management. Curr Urol Rep 8: 83-88, 2007.

3 Safwat AS, Bissada NK, Seyam RM, Al Sobhi S and Hanash KA: The clinical spectrum of phaeochromocytoma: analysis of 115 patients. BJU Int 101: 1561-1564, 2008.
4 Cunningham SC, Suh HS, Winter JM, Montgomery E, Schulick $\mathrm{RD}$, Cameron JL and Yeo CJ: Retroperitoneal paraganglioma: single-institution experience and review of the literature. J Gastrointest Surg 10: 1156-1163, 2006.

5 Woods MS, Mullins JR, Shellito JL, Uhlig PN and Brinton EH: Recommendations concerning the operative approach for pheochromocytoma invading the inferior vena cava. Surgery 115: 771-775, 1994.

6 Waidelich R, Weninger E, Denzlinger C, Müller-Lisse U, Schmeller $\mathrm{H}$ and Schmeller N: Use of veno-venous bypass for resection of malignant pheochromocytoma with vena caval thrombus. Int Urol Nephrol 34: 241-243, 2002.

7 Van Slycke S, Caiazzo R, Pigny P, Cardot-Bauters C, Arnalsteen L, D'Herbomez M, Leteurtre E, Rouaix-Emery N, Ernst O, Huglo D, Vantyghem MC, Wemeau JL, Carnaille B and Pattou F: Local-regional recurrence of sporadic or syndromic abdominal extra-adrenal paraganglioma: incidence, characteristics, and outcome. Surgery 146: 986-992, 2009.

8 Parnaby CN, Serpell MG, Connell JM and O'Dwyer PJ: Perioperative haemodynamic changes in patients undergoing laparoscopic adrenalectomy for phaeochromocytomas and other adrenal tumors. Surgeon 8: 9-14, 2010.

9 Facciuto ME, Singh MK, Rocca JP, Rochon C, Rodriguez Davalos MI, Schwalb DM, Choudhury M and Sheiner PA: Benefits of liver transplantation surgical techniques in the management of extensive retroperitoneal tumors. World J Surg 32: 2403-2407, 2008.

10 Fusai G, Steinberg R, Prachalias A, Heaton ND, Spitz L and Rela M: Ex vivo liver surgery for extraadrenal pheochromocytoma. Pediatr Surg Int 22: 282-285, 2006.

11 Delis SG, Bakogiannis A, Ciancio G and Soloway M: Surgical management of large adrenal tumors: the University of Miami experience using liver transplantation techniques. BJU int 102: 1394-1399, 2008.

12 Bourke CJ, Lynch S, Irving H, Borzi PA: Retroperitoneal paraganglioma in a child: resection and vena caval reconstruction. Pediatr Surg Int 18: 505-508, 2002.

13 Tominaga M, Ku Y, Iwasaki T, Muramatsu S, Kuroda Y, Shima Y, Takao Y and Obara H: Resection of pheochromocytoma under inferior vena cava isolation and extracorporeal charcoal hemoperfusion. Arch Surg 133: 1016-1018, 1998.
Received October 28, 2016

Revised November 14, 2016

Accepted November 15, 2016 